\title{
Estimação de parâmetros cinéticos do modelo de transferência de massa da cromatografia em coluna empregando o método de Monte Carlo com cadeias de Markov
}

\author{
Josiele da Silva Teixeira ${ }^{1}$ \\ Leonardo Tavares Stutz ${ }^{2}$ \\ Diego Campos Knupp ${ }^{3}$ \\ Antônio José da Silva Neto ${ }^{4}$
}

Departamento de Engenharia Mecânica e Energia, IPRJ, UERJ, Nova Friburgo RJ

Mirtha Irizar Mesa ${ }^{5}$

Orestes Llanes Santiago 6

Departamento de Automática y Computación, Fac. Eléctrica, ISPJAE, La Habana, Cuba

\begin{abstract}
Resumo. O presente trabalho apresenta um estudo referente à aplicação da abordagem Bayesiana como técnica de solução do problema inverso de estimação de parâmetros em processos de cromatográficos de separação da glicose e frutose presentes no suco de caju. O problema direto é formulado considerando-se a abordagem "Front Velocity", que considera a convecção como a fase dominante no transporte de soluto ao longo da coluna cromatográfica. A solução do problema inverso de estimação, é obtida empregando o Método de Monte Carlo com cadeias de Markov (MCMC), implementado através do algoritmo de MetropolisHastings.
\end{abstract}

Palavras-chave. Inferência Bayesiana, Cadeia de Markov, Cromatografia.

\section{Introdução}

A necessidade de separar compostos presentes em misturas, seja para qualificá-los ou quantificá-los, vem desde o início da química analítica, mas com o passar do tempo essa necessidade se estendeu á diversas áreas além da química. Devido a grande importância econômica e social que muitos compostos possuem, diversos setores da indústria, tais como petroquímico, alimentício, químico, farmacêutico, dentre outros, tem buscado desenvolver

\footnotetext{
1 josyelly1@gmail.com

${ }^{2}$ ltstutz@iprj.uerj.br

${ }^{3}$ diegoknupp@gmail.com

${ }^{4}$ ajsneto@iprj.uerj.br

${ }^{5}$ mirtha@electrica.cujae.edu.cu

${ }^{6}$ orestes@electrica.cujae.edu.cu
} 
e aperfeiçoar técnicas de separação de compostos [2]. Este trabalho tem como objetivo estimar as constantes cinéticas de adsorção e dessorção do processo de transferência de massa durante o processo de separação em Leito Móvel Simulado (LMS), considerando uma nova abordagem denominada "Front Velocity" para descrever o processo de transferência de massa [2]. O problema inverso de estimação de parâmetros é resolvido a partir da abordagem Bayesiana, que tem sido bastante utilizada como ferramenta para resolver problemas de estimação de parâmetros, onde as grandezas do problema são modeladas como variáveis aleatórias. O método de Monte Carlo com cadeias de Markov (MCMC), implementado através do algoritmo de Metropolis-Hastings é utilizado para simular amostras da distribuição de probabilidade a posteriori de interesse.

\section{Formulação do Problema Direto em Cromatografia}

Neste trabalho, a transferência de massa que ocorre durante o processo de separação, foi representada utilizando uma nova abordagem denominada "Front Velocity" [3]. Esta abordagem estabelece que a convecção é a fase dominante no transporte de soluto ao longo da coluna cromatográfica. Este é um modelo discreto (etapas) onde a vazão determina o avanço da fase líquida ao longo da coluna.

Para descrever o comportamento dos sítios de adsorção das colunas cromatográficas, dois diferentes mecanismos cinéticos foram adotados. No primeiro (Eq. 1) foi assumida uma relação linear, onde não existe uma capacidade máxima de adsorção $q_{m}$, enquanto que no segundo caso uma equação cinética (Eq. 2) que contempla a saturação das colunas foi utilizada.

$$
\begin{aligned}
& r_{q}=\kappa_{a d s} C-\kappa_{\text {des }} q \\
& r_{q}=\kappa_{a d s} C\left(q_{m}-q\right)-\kappa_{\text {des }} q
\end{aligned}
$$

onde, $C$ é a concentração na fase líquida, $q_{m}$ é a capacidade máxima de adsorção da fase sólida, $q$ é a concentração na fase sólida, $\kappa_{a d s}$ é a constante cinética de adsorção e $\kappa_{\text {des }}$ é a constante cinética dessorção.

\section{Problema Inverso e Inferência Bayesiana}

O problema inverso em cromatografia têm como objetivo estimar as constantes cinéticas de adsorção e dessorção desconhecidas do modelo de transferência de massa adotado. No entanto, pode-se estimar também a constante de equilíbrio $\left(k_{e q}=k_{a d s} / k_{d e s}\right)$.Dessa forma, será considerado neste trabalho dois casos de estudo. No Caso I as constantes cinéticas de adsorção e de dessorção para cada substância (frutose e glucose) constitui os parâmetros a serem estimados e neste caso será considerando a isoterma linear dada pela Equação 1, enquanto que, no Caso II, têm-se como objetivo estimar as constantes cinéticas equilíbrio e dessorção para cada substância e será utilizada a isoterma de Langmuir dada pela Equação 2 .

Do ponto de vista Bayesiano, a solução do problema inverso, dadas as observações experimentais a posteriori $\boldsymbol{Y}_{\text {exp }}$, é uma função de densidade de probabilidade de $\boldsymbol{\kappa}$, denotada 
por $P_{\text {post }}\left(\boldsymbol{\kappa} \mid \boldsymbol{Y}_{\text {exp }}\right)$, que pode ser escrita, de acordo com o teorema de Bayes, como [4]

$$
P_{\text {post }}\left(\boldsymbol{\kappa} \mid \boldsymbol{Y}_{\text {exp }}\right)=\frac{P\left(\boldsymbol{Y}_{\text {exp }} \mid \boldsymbol{\kappa}\right) P_{p r}(\boldsymbol{\kappa})}{P\left(\boldsymbol{Y}_{\text {exp }}\right)}
$$

onde $P_{p r}(\boldsymbol{\kappa})$ é a informação a priori das variáveis, modelada como uma distribuição de probabilidade, $P\left(\boldsymbol{Y}_{\text {exp }} \mid \boldsymbol{\kappa}\right)$ é a função de verossimilhança que representa a probabilidade de ocorrência dos dados experimentais $\boldsymbol{Y}_{\text {exp }}$ dados os parâmetros do modelo $\boldsymbol{\kappa}$ e $P\left(\boldsymbol{Y}_{\text {exp }}\right)$ é a densidade marginal, que funciona como uma constante de normalização.

Técnicas de amostragem são muito utilizadas quando a distribuição a posteriori não pode ser obtida através de expressão analítica ou simulação numérica. Neste trabalho amostras foram obtidas através dos métodos de Monte Carlo via Cadeias de Markov (MCMC), cuja ideia principal é simular amostras aleatórias no domínio do parâmetro $\boldsymbol{\kappa}$, de tal forma, que convirjam para a distribuição a posteriori $P_{\text {post }}\left(\boldsymbol{\kappa} \mid \boldsymbol{Y}_{\text {exp }}\right)$, utilizando técnicas de simulação iterativa, baseadas em cadeias de Markov.

Algoritmos específicos são utilizados para a obtenção das cadeias de Markov, onde neste trabalho foi utilizado o algoritmo de Metropolis-Hastings, que faz uso de uma função densidade de probabilidade auxiliar $\mathbf{q}$, da qual seja fácil se obter valores amostrais. Para mais detalhes da metodologia adotada consultar [4].

\section{Resultados e discussões}

As simulações foram realizadas baseadas na cromatografia de adsorção da glicose e frutose presentes no suco de caju, utilizando dados pseudos experimentais, referentes a um pulso de concentração de uma amostra de $300 \mathrm{ml}$ injetado em uma unidade de LMS com 11 colunas cromatográficas dispostas em série [1]. Além Disso, para cada caso de estudo dois níveis de ruído foram adicionados aos dados pseudos experimentais, um mais brando, $\boldsymbol{p}=0.02$, Caso Ia, e um mais intenso, $\boldsymbol{p}=0.05$, Caso $\mathrm{Ib}$, equivalentes à $2 \%$ e $5 \%$, respectivamente. O sinal ruidoso foi obtido a partir da relação $\boldsymbol{Y}_{\text {exp }, i}=\boldsymbol{Y}_{i} \pm$ p.rand. $(\max (\boldsymbol{Y})-\min (\boldsymbol{Y}))$.

\subsection{Resultados para o Caso I}

Para todas as cadeias foram utilizados 10.000 estados, descartando as 3.000 amostras iniciais. A partir de uma análise qualitativa das Figuras 1 e 2, pode-se observar que para ambos níveis de ruído todas as cadeias apresentadas convergiram a partir de 2000 estados aproximadamente. Ao analisar quantitativamente a Tabela 1, verifica-se que, para o Caso Ia, os valores amostrados possuem desvios menores e consequentemente médias mais próximas do valor exato quando comparados com os desvios e médias obtidos no Caso Ib, este resultado concorda com o fato de que dados experimentais com um nível de ruído mais intenso conduzem a estimação de valores com um nível de incerteza maior. Outra consequência desde fato é a obtenção de intervalos de confiança maiores para o Caso Ib. 


\subsection{Resultados para o Caso II}

Neste caso, foram utilizadas cadeias com 10.000 estados, descartando as 4.000 amostras iniciais. Para o Caso II, pode-se observar que, com exceção da cadeia da constante de dessorção no Caso IIa, as demais cadeias apresentam convergência a partir de 2.000 estados aproximadamente. Para ambos níveis de ruído as cadeias obtidas para a constante de dessorção apresentaram um comportamento mais oscilatório, tal comportamento foi mais acentuado para o Caso IIb, devido a utilização de desvio maior implicando assim em um espaço de busca mais amplo. Os resultados apresentados na Tabela 2, mostram que no Caso IIa foram obtidos menores desvios, médias mais próximas dos valores exatos e também intervalos de confiança menores, em relação ao Caso IIb.

Figura 1: Cadeias de Markov - Caso Ia (2 \%)

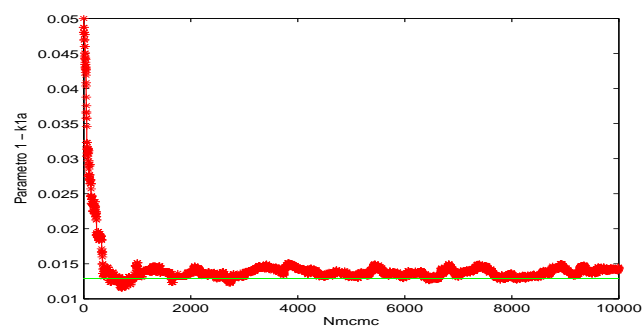

(a) $k_{a d s, G}$

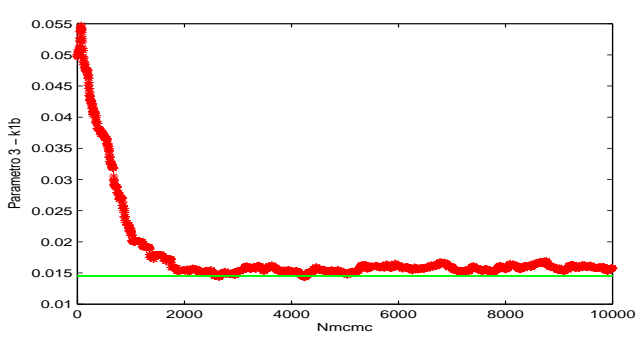

(c) $k_{a d s, F}$

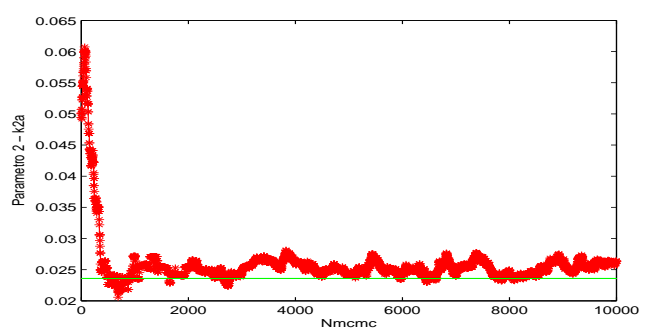

(b) $k_{d e s, G}$

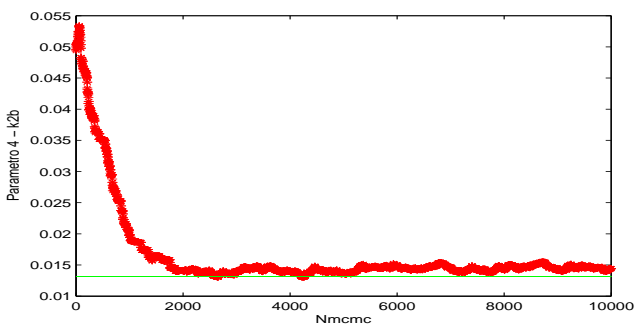

(d) $k_{d e s, F}$

Tabela 1: Resumo dos resultados obtidos para o caso Ia

\begin{tabular}{cccccc}
\hline & & I.C $(99 \%)$ & Exato & Média & D.P \\
\hline \multirow{3}{*}{$2 \%$} & $k_{\text {ads }, G}$ & {$[0,01242 ; 0,01362]$} & 0.01292 & 0.01301 & 0.000298140 \\
& $k_{\text {des }, G}$ & {$[0,02269 ; 0,02501]$} & 0.02362 & 0.02383 & 0.000561240 \\
& $k_{\text {ads }, F}$ & {$[0,01423 ; 0,01494]$} & 0.01452 & 0.01455 & 0.000151987 \\
& $k_{\text {des }, G}$ & {$[0,01288 ; 0,01358]$} & 0.01318 & 0.01319 & 0.000146307 \\
\hline \multirow{4}{*}{$5 \%$} & $k_{\text {ads }, G}$ & {$[0,01270 ; 0,01498]$} & 0.01292 & 0.01372 & 0.000534907 \\
& $k_{\text {des }, G}$ & {$[0,02329 ; 0,02752]$} & 0.02362 & 0.02518 & 0.000997769 \\
& $k_{\text {ads }, F}$ & {$[0,01473 ; 0,01686]$} & 0.01452 & 0.01586 & 0.000403191 \\
& $k_{\text {des }, G}$ & {$[0,01357 ; 0,01545]$} & 0.01318 & 0.01452 & 0.000383235 \\
\hline
\end{tabular}


Figura 2: Cadeias de Markov - Caso Ib (5 \%)

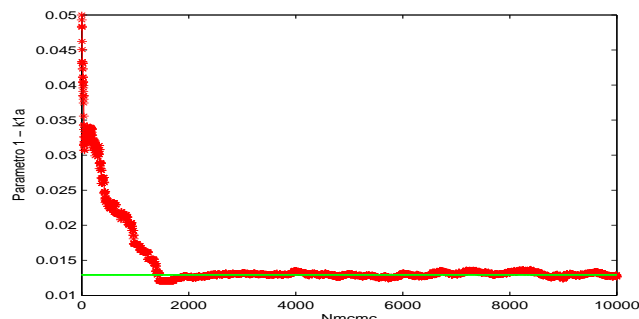

(a) $k_{a d s, G}$

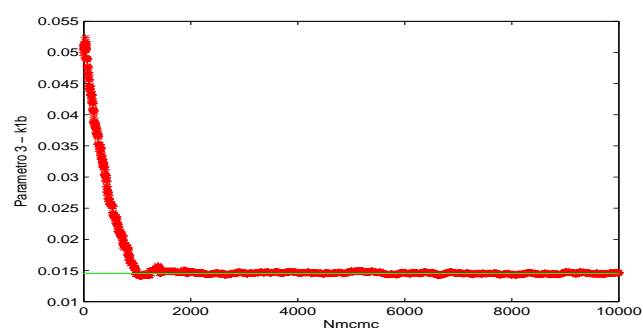

(c) $k_{a d s, F}$

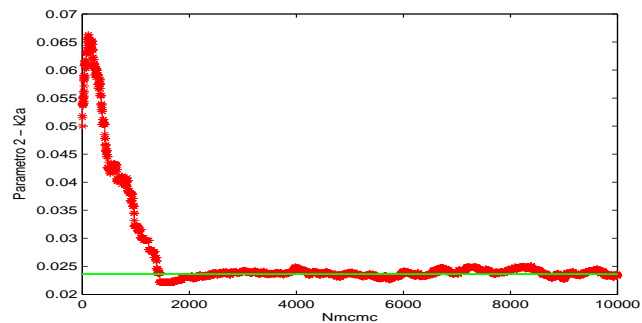

(b) $k_{\text {des }, G}$

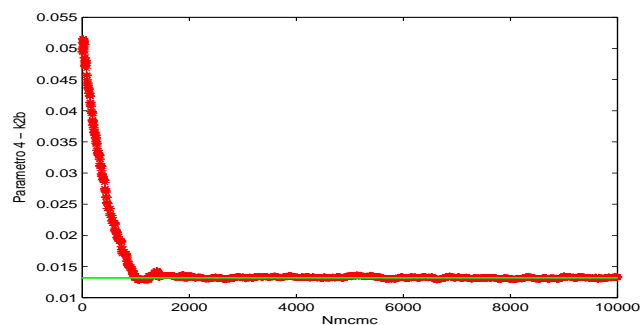

(d) $k_{\text {des }, F}$

Figura 3: Cadeias de Markov - Caso IIa (2 \%)

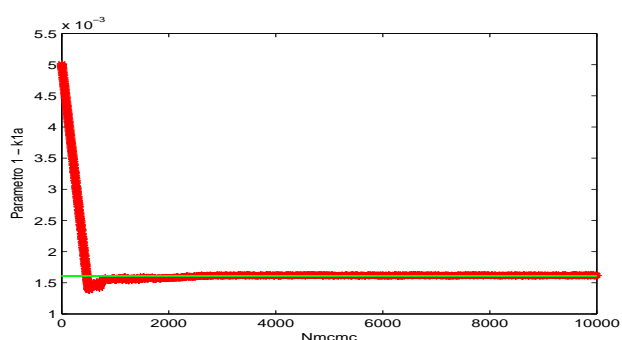

(a) $k_{e q, G}$

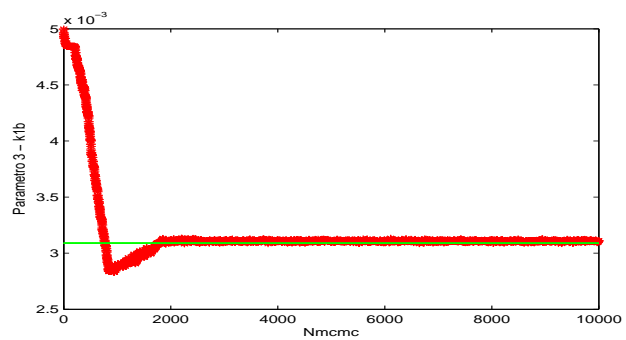

(c) $k_{e q, F}$

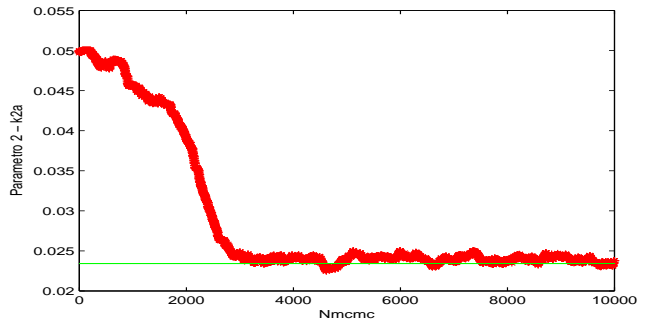

(b) $k_{d e s, G}$

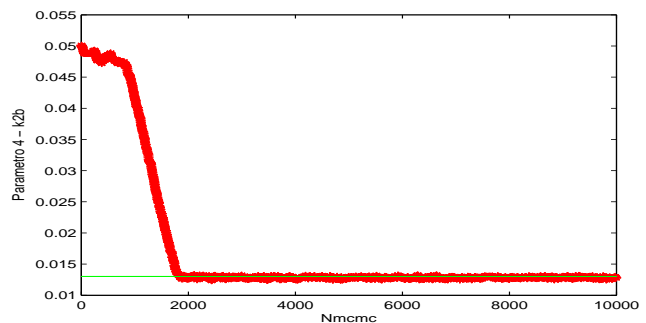

(d) $k_{d e s, F}$ 
Figura 4: Cadeias de Markov - Caso IIb (5 \%)

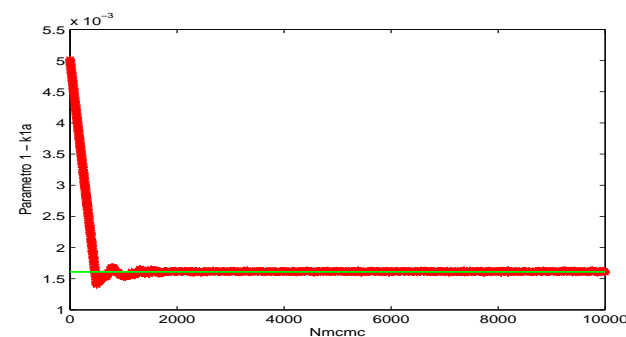

(a) $k_{e q, G}$

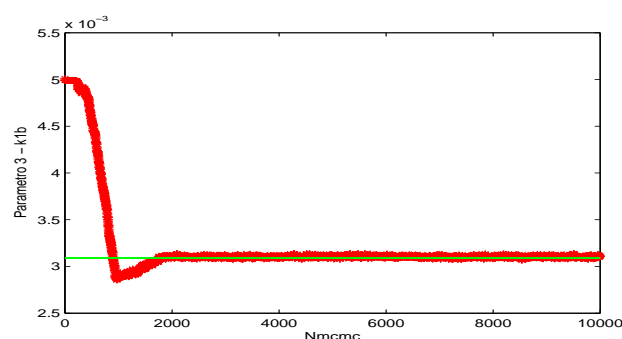

(c) $k_{e q, F}$

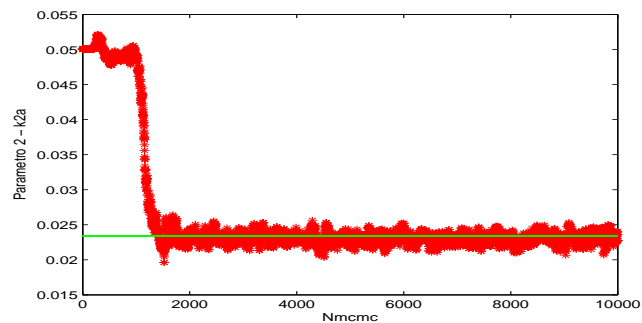

(b) $k_{\text {des }, G}$

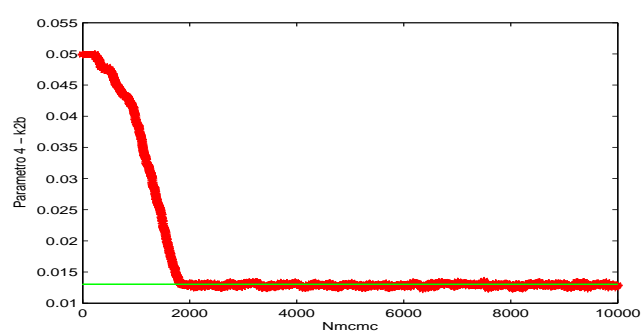

(d) $k_{d e s, F}$

Tabela 2: Resumo dos resultados obtidos para o caso IIa

\begin{tabular}{cccccc}
\hline & I.C $(99 \%)$ & Exato & Média & D.P & \\
\hline & $k_{\text {eq }, G}$ & {$[0,001592 ; 0,001638]$} & 0.00161 & 0.001616 & 0.000004920 \\
$2 \%$ & $k_{\text {des }, G}$ & {$[0,021209 ; 0,024916]$} & 0.02339 & 0.023023 & 0.000408550 \\
& $k_{\text {eq }, F}$ & {$[0,003077 ; 0,003133]$} & 0.00309 & 0.003103 & 0.000006950 \\
& $k_{\text {des }, G}$ & {$[0,012301 ; 0,013463]$} & 0.01304 & 0.012901 & 0.000149949 \\
\hline \multirow{4}{*}{$5 \%$} & $k_{\text {eq, },}$ & {$[0,001604 ; 0,001631]$} & 0.00161 & 0.001617 & 0.000009345 \\
& $k_{\text {des }, G}$ & {$[0,023029 ; 0,024988]$} & 0.02339 & 0.024019 & 0.000769834 \\
& $k_{\text {eq, }, F}$ & {$[0,003089 ; 0,003126]$} & 0.00309 & 0.003107 & 0.000010855 \\
& $k_{\text {des }, G}$ & {$[0,012470 ; 0,013214]$} & 0.01304 & 0.012824 & 0.000235370 \\
\hline
\end{tabular}




\section{Conclusões}

O presente trabalho teve como objetivo principal a aplicação da abordagem Bayesiana na como metodologia de solução do problema inverso de estimação de parâmetros no problema de cromatografia da glicose e frutose presentes no suco de caju. Apresentouse uma formulação do problema direto considerando-se a abordagem "Front Velocity", cuja solução foi obtida através do método Euler. Considerou-se ainda dois modelos de transferência de massa, o modelo Linear e de Langmuir. A solução do problema inverso de estimação, foi obtida pelo Método de Monte Carlo com cadeias de Markov (MCMC), implementado através do algoritmo de Metropolis-Hastings. O método de MCMC se apresentou eficiente resolver os casos estudados, onde os dados experimentais possuíam diferentes níveis de incerteza associado, sendo assim capaz de estimar os parâmetros com a acurácia requerida para o problema, demonstrando assim uma de suas grande vantagens que é tratar de forma adequada as incertezas inerentes ao problema, o que para muitos outros métodos de estimação esse é um problema a mais.

\section{Agradecimentos}

Os autores agradecem o apoio financeiro da FAPERJ, Fundação Carlos Chagas Filho de Amparo à Pesquisa do Estado do Rio de Janeiro e da CAPES, Coordenação de Aperfeiçoamento de Pessoal de Nível Superior.

\section{Referências}

[1] D. C. S. Azevedo and A. Rodrigues, SMB chromatography applied to the separation/purification of fructose from cashew apple juice, Brazilian Journal of Chemical Engineering, vol. 17, 507-516, (2000).

[2] A. J. Bihain, A. J. Silva-Neto, O. Llanes-Santiago, J. C. Afonso and L. D. Câmara, The front velocity modelling approach in the chromatographic column characterization of glucose and fructose separation in SMB, Trends in Chromatography, vol. 7, 33-41, (2012).

[3] L.D.T.Câmara, Chromatographic Columns Charaterization for SMB (Simulated Moving Bed) Separation of Gluose and Frutose, Proceeding Series of the European Congress of Chemical Engineering, vol. 8, (2011).

[4] J.P. Kaipio and E. Somersalo, Statistical and Computational Inverse Problems, Applied Mathematical Sciences 160, Spring-Verlag, vol. 1, (2004).

[5] J. S. Teixeira, D. C. Knupp, L. T. Stutz e A. J. Silva Neto, Identificação de Danos Estruturais via Método de Monte Carlo com Cadeia de Markov, Anais do Encontro de Modelagem Computacional, vol. 16, (2013). 